\title{
TAGUNG
}

\section{Sportpolitik in Europa: Mehr als die Debatte um Ronaldos Frisur?}

\author{
Frédéric Krumbein ${ }^{*}$
}

\section{Vielfalt der Disziplinen, Perspektiven und Akteure in der europäischen Sportwissen- schaft}

Das Ziel der Tagung bestand nach Jürgen Mittag in dem Austausch zwischen den Disziplinen, verschiedener Akteure sowie von Praxis und Wissenschaft über das Themenfeld ,europäische Sportpolitik“. Forschung über Europa stelle immer eine Integrationswissenschaft im Sinne der Integration verschiedener Perspektiven und Disziplinen dar. Die Europäische Union beeinflusse fast alle Gebiete der Politik, auch den Bereich des Sports.

Ralf Kleinfeld stellte anschließend fest, dass es in der politikwissenschaftlichen Europaforschung verschiedene Zugänge gibt: von der Teildisziplin der internationalen Beziehungen bis hin zur vergleichenden Politikwissenschaft und der Analyse des europäischen Mehrebenensystems.

Christoph Breuer sprach über wirtschaftswissenschaftliche Zugänge zum Themenfeld der europäischen Sportpolitik. Aus Sicht der Wirtschaftswissenschaften seien in der europäischen Sportpolitik Kosten und Nutzen unterschiedlicher Alternativen abzuwägen. Es werde weiterhin untersucht, wie Akteure unter Knappheitsbedingungen handeln. Die ökonomische Logik der Sportsysteme stelle den typischen Analyserahmen dar. Beispielsweise untersuche die ökonomisch ausgerichtete Sportwissenschaft wie die Chancengleichheit zwischen den Sportvereinen großer und kleiner Staaten sichergestellt werden könne.

\section{Auf dem Weg zur europäischen Sportpolitik}

Eine Tagung der Deutschen Sporthochschule Köln, des Instituts für europäische Sportentwicklung und Freizeitforschung, der Europäischen Akademie des Sports und des Arbeitskreises Europäische Integration e. V.

Die Tagung wurde von der Europäischen Kommission finanziell unterstützt.

Köln, 28.-29. Juni 2012

\section{Begrüßung}

Prof. Dr. Walter TOKARSKI, Rektor Deutsche Sporthochschule Köln

Einführung, Zugänge und theoretischkonzeptionelle Perspektiven

Einführung und Vorsitz

Prof. Dr. Jürgen MITTAG, Deutsche Sporthochschule Köln

Politikwissenschaftliche Zugänge

Prof. Dr. Ralf KLEINFELD, Universität Osnabrück

Rechtswissenschaftliche Zugänge

Martin NOLTE, Deutsche Sporthochschule Köln

Ökonomische Zugänge

Christoph BREUER, Deutsche Sporthochschule Köln

Kommunikationswissenschaftliche Zugänge Roger BLUM, Universität Bern

* Dr. Frédéric Krumbein, Geschäftsführer des Arbeitskreises Europäische Integration e.V., Berlin. 
Anschließend referierte Martin Nolte über die rechtswissenschaftliche Perspektive auf die europäische Sportpolitik. Der Sport ruhe auf den beiden Säulen des zwischenstaatlichen Rechts und des selbstgesetzten Rechts der Sportverbände.

Seit dem Jahr 2006 dehne sich die Anwendbarkeit des EU-Rechts immer weiter auf den Sport aus. Eines der bekanntesten Beispiele stelle das „Bosman-Urteil““1 aus dem Jahr 1995 dar, welches die Arbeitnehmerfreizügigkeit auf den Sport angewandt habe. Sportwetten und Anti-Dopingmaßnahmen würden ebenfalls durch europäisches Recht reguliert. Die zentrale Vermarktung der Fernsehrechte unterliege ebenfalls dem europäischen Kartellrecht.

Die Förderung des Sports sei in Art. 165 des Vertrags über die Arbeitsweise der Europäischen Union (AEUV) festgeschrieben. Im deutschen Grundgesetz bestehe hingegen eine rechtliche Lücke bei der Sportförderung. Der Sport werde jährlich mit 500 Millionen Euro vom Staat gefördert, ohne dass diese ein Staatsziel darstelle. Der Art. 165 AEUV könne diese Lücke bis zu einem gewissen Grad schließen.

Die Europäische Union verfüge außerdem über Zuständigkeiten bei der Unterstützung, Koordination und Ergänzung der mitgliedstaatlichen Sportpolitik, beispielsweise bei der Bekämpfung von Spielabsprachen. Des Weiteren verfügen Sportveranstaltungen nach EU-Recht über einen originären Charakter, vergleichbar mit künstlerischen und musikalischen Veranstaltungen, die urheberrechtlich geschützt werden könnten. So könne untersagt werden, dass Zuschauer Sportveranstaltungen filmen und diese ohne Zustimmung der Veranstalter verbreiten. Aktuelle Trends bestünden in der weiteren Ausdehnung der Geltung des europäischen Sportrechts und dessen stärkerer Kodifikation.

Die kommunikations- und medienwissenschaftlichen Zugänge zur europäischen Sport-
Entwicklungslinien des Sports und der Sportforschung auf europäischer Ebene zwischen „Konstitutionalisierung“ und Europäisierung

Vorsitz

Sven GÜLDENPFENNIG, ehemaliger Leiter des Deutschen Olympischen Instituts, Aachen

Anfänge und Ausgestaltung europabezogener Sportforschung an Universitäten und Forschungsinstituten

Karen PETRY, Deutsche Sporthochschule Köln

Die Entwicklung europäischer Sportpolitik und -forschung

Jacob KORNBECK, Generaldirektion Bildung und Sport, Europäische Kommission, Brüssel

Die Sportpolitik von Europäischer Gemeinschaft und Europäischer Union

Stephan KLAUS, Universität Osnabrück

Die Europäisierung des Fußballs

Alexander BRAND und Arne NIEMANN, Johannes Gutenberg Universität Mainz

Akteure im Prozess europäischer

Willensbildung und Entscheidungsfindung

Vorsitz

Georg ANDERS, Deutsche Sporthochschule Köln

Die Rolle des Europarats in der Sportpolitik und die europ. Dachverbände: Das Beispiel des Europäischen Paralympischen Komitees

Matthias GUETT, European Paralympic Committee, Wien

Sportpolitik im Mehrebenensystem: Die Rolle der Bundesländer und der SMK

Stefan CHRISTMANN, Ministerium des Innern und für Sport, Mainz

Europäische Sportpolitik zwischen Government und Governance?

Hans BRUYNINCKX, Katholieke Universiteit Leuven

20 Jahre Institut für Europäische Sportentwicklung und Freizeitforschung: Bausteine zur Erforschung europäischer Sportpolitik

Walter TOKARSKI, Rektor, Deutsche Sporthochschule Köln

1 EuGH Rs. C-415/93 (ASBL/Bosman), Slg. 1995, S. I-4921. 
politik wurden von Roger Blum vorgestellt. Die Kommunikationswissenschaft befasse sich in erster Linie mit Massenmedien und damit auch mit der europäischen Öffentlichkeit. Öffentlichkeit stelle zum einen den Raum dar, der vom privaten Raum abgegrenzt werde. Zum anderen sei Öffentlichkeit ein Prinzip, welches sich vom Geheimen unterscheide. Sie bestehe ferner aus einer Vielzahl von Kommunikationsformen, zu denen der Zugang offen sei und an dem sich verschiedene Akteure beteiligten.

Die europäische Öffentlichkeit verfüge über mehrere typische Charakteristika: unterschiedliche Kulturen, unterschiedliche Interessen und unterschiedliche Sprachen. Es gebe kaum europäische Medien. Diese scheiterten an der fehlenden , lingua franca'. Menschen nutzten auch kaum fremdsprachige Medien. Die Berichterstattung über Europa genieße eher eine niedrige Priorität in der Berichterstattung nationaler Medien. Die europäische Öffentlichkeit funktioniere eher über Ereignisse und Themen und stelle nur eine Teilöffentlichkeit dar. Das Publikum der europäischen Öffentlichkeit bestehe nur aus einem kleinen Teil der Bevölkerung, wie den Politikinteressierten. Durch die Ereignisbezogenheit werde europäische Öffentlichkeit nur punktuell geschaffen.

Schließlich vertrat Blum die These, dass die sportliche Öffentlichkeit und die Unterhaltungsöffentlichkeit in Europa bedeutender seien als die politische Öffentlichkeit. Dies zeige sich an der Popularität von Ereignissen wie der Fußball-Europameisterschaft oder dem Eurovision Song Contest.

Prozesse der Europäisierung am Beispiel der Sportwissenschaft und des Fußballs

Karen Petry berichtete über die Vernetzung der Sportwissenschaft in Europa und deren Charakteristika. Als ein Netzwerk europäischer Sportforschung sei im Jahr 1989 das ,European Network of Sport Science, Education \&
Öffentliche Veranstaltung und

Podiumsdiskussion: Die EURO 2012: Politik statt Sport?

Moderation

Jochen LEUFGENS, WDR, Köln

Diskutanten

Folker HELLMUND, Leiter EU-Büro, European Olympic Committee, Brüssel

Thomas HORKY, Macromedia Hochschule für Medien und Kommunikation, Hamburg

Petra KAMMEREVERT, MdEP, Mitglied im Ausschuss für Kultur und Bildung, Brüssel

Jacob KORNBECK, Generaldirektion Bildung und Sport, Europäische Kommission, Brüssel

Olaf SUNDERMEYER, Journalist, Berlin

Problem- und Interaktionsfelder europäischer Sportpolitik

Vorsitz

Ben WEINBERG, Deutsche Sporthochschule Köln Die sozialpolitischen Bestimmungen des LissabonVertrags und die Auswirkungen auf den Sport

Prof. Dr. h.c. A. Walfried KÖNIG, Deutsche Sporthochschule Köln

Dopingpolitik zwischen nationaler und europäischer Ebene

Henk Erik MEIER, Universität Münster

Entwicklung und Bedeutung der EU-Guidelines for Physical Activity

Roland NAUL, Universität Duisburg-Essen

Die finanzielle Dimension europäischer Sportpolitik

Daniel DICKMANN, Universität Potsdam

Bedeutung der Sportgroßereignisse

Vorsitz

Michael GROLL, Deutsche Sporthochschule Köln Die Fußball-Europameisterschaften: Vom Nebenschauplatz zum Sportgroßereignis

Benjamin LEGRAND, Ruhr-Universität Bochum

EURO und Champions League: Wegbereiter transnationaler Sportpolitik?

Jörg-Uwe NIELAND, Deutsche Sporthochschule Köln

Fans zwischen Identitätssuche und (Anti-)Diskriminierung

Gerd DEMBOWSKI, Freie Universität Berlin

Zusammenfassung, Bilanz und Ausblick

Prof. Dr. Jürgen MITTAG, Deutsche Sporthochschule Köln 
Employment ${ }^{`}$ gegründet worden. Dieses Netzwerk sportwissenschaftlicher Institute habe früh angefangen mit der Europäischen Kommission zu kooperieren und sich für eine Förderung der Sportwissenschaft durch die Europäische Union einzusetzen. Alle zwei Jahre fänden Treffen der Mitglieder des Netzwerkes, zuletzt in Paris im Jahr 2011, statt. Daneben existierten weitere Vereinigungen, die sich mit Sportpolitik auf europäischer Ebene beschäftigten.

Die europabezogene Sportforschung lasse sich grob in drei Bereiche unterteilen: international vergleichende Länderstudien, Studien zur direkten und indirekten Sportpolitik und sportrechtliche Studien. Die fußballbezogene Europaforschung habe einen prominenten Stellenwert innerhalb der Sportwissenschaft eingenommen. Der Fußball zeige seine Dominanz, die er im Sport ausübe, auch in der sportwissenschaftlichen Forschung.

Die weitere Entwicklung der europäischen Sportpolitik und -forschung wurde von Jacob Kornbeck skizziert. Er stellte zu Beginn die Frage, ob die europäische Integration oder die Forschung zur europäischen Integration zuerst existierten. Diese Frage erscheine nur im ersten Moment paradox. So habe es schon Integrationstheorien Anfang der 1950er Jahre gegeben, noch vor der Gründung der Europäischen Gemeinschaft für Kohle und Stahl.

Ein Indikator für die zunehmende Bedeutung des Themas Sport in der Europäischen Union stelle die Steigerung der Zahl der Publikationen über sportpolitische Themen in der Zentralbibliothek der Europäischen Kommission dar. Publikationen über Doping und Fußball seien ebenfalls angestiegen. Der Onlinekatalog der Zentralbibliothek der Europäischen Kommission stelle eine gute Quelle für EU-Dokumente dar, auch im Bereich der europäischen Sportpolitik. $^{2}$

Der Europäische Gerichtshof und seine Urteile seien eine zentrale Triebkraft der europäischen
Sportwissenschaft gewesen, stärker als bei anderen Themenfeldern der europäischen Integration. Seine Eingangsfrage beantwortete Kornbeck dergestalt, dass die europäische Integration seiner Ansicht nach vor der Integrationsforschung existiert habe.

Jürgen Mittag sprach ebenfalls über die europäische Sportpolitik. Die Europäische Union habe sich zu Beginn nur zurückhaltend in der Sportpolitik betätigt. Deren Bedeutung sei dann kontinuierlich gestiegen: In einer Phase der Identitätsfindung der Europäischen Union sei Sport als Instrument zur Schaffung einer europäischen Identität benutzt worden; und mit dem „Bosman-Urteil“ von 1995 habe der Europäische Gerichtshof den gemeinsamen Binnenmarkt auf den Bereich des Sports ausgedehnt. Seit den 1990er Jahren verlagere sich der Sport als Politikfeld zunehmend auf die europäische Ebene. In den 1980er Jahren sei der Sport noch sehr national geprägt gewesen. Die jüngsten Entwicklungen stellten die Regelungsautonomie der Sportverbände zunehmend infrage. Sportpolitik werde immer mehr supranational ausgestaltet.

Arne Niemann und Alexander Brand referierten über die Europäisierung des Fußballs. Europäisierung könne in zwei Stränge geteilt werden: „downloading“, das heißt, die Initiierung von Prozessen der Europäisierung auf der Ebene der politischen Institutionen der Europäischen Union; und „uploading“, das heißt die Initiierung von Europäisierung durch nationale Regierungen. Parallel dazu liefen transnationale Verflechtungsprozesse ab (,crossloading“). Der Begriff der Europäisierung könne außerdem eng oder weit definiert werden. Eine enge Definition beziehe Europäisierung nur auf die Politiken der Europäischen Union. Eine weite Definition umfasse alle Anpassungsprozesse und Transformationsdynamiken der europäischen Integration.

Ein Beispiel für „downloading“ sah Brand im „Bosman-Urteil“. Der Europäische Gerichts-

2 Vgl. http://ec.europa.eu/eclas/F (letzter Zugriff: 21.9.2012). 
hof habe beim „Bosman-Urteil“ entschieden, dass Restriktionen hinsichtlich der Anzahl EUausländischer Spieler in Vereinsmannschaften unzulässig seien.

Das Urteil habe zu einer tiefgreifenden Veränderung der Zusammensetzung der Profimannschaften im Fußball geführt und das Primat des EU-Rechts vor der Selbstregulierung der Fußballverbände verdeutlicht. Im Spielermarkt der ersten und zweiten Bundesliga gäbe es nur noch etwa 50 Prozent Deutsche, vor dem Urteil seien es noch etwa 80 Prozent gewesen. Als Konsequenz werde der fußballerische Nachwuchs stärker gefördert, damit es hinreichend viele gute Fußballspieler für die deutsche $\mathrm{Na}$ tionalmannschaft gebe.

In Italien und Österreich habe das „BosmanUrteil" ebenfalls zu einer Erhöhung des Anteils ausländischer Spieler in den Ligen geführt (circa 40 Prozent). Der Spielermarkt in Frankreich habe sich nach Ansicht von Brand hingegen nur wenig verändert: Dieser sei traditionell offen gewesen.

Die Gründung der Champions League im Fußball interpretiert Niemann als einen Prozess des ,crossloading“. Für die nationalen Proficlubs stelle die Teilnahme an der Champions League aus finanziellen Gründen und aus Prestigegründen einen starken Anreiz dar. Die großen Proficlubs orientierten sich an der europäischen Konkurrenz. Der Vergleichsrahmen werde europäisch.

Abschließend stellte Brand dar, dass es Prozesse der Europäisierung auch auf der Ebene der Fans gebe. So zeige sich, dass die Europäer Lieblingsclubs haben. Der beliebteste Club in Europa sei der FC Barcelona, mit deutlichem Abstand gefolgt von Real Madrid. Die Champions League verfüge ebenfalls über einen sehr hohen Stellenwert unter den Fußballfans. $\mathrm{Zu}$ Beginn der Champions League sei die Reputation noch längst nicht so ausgeprägt gewesen. Prozesse der Europäisierung könnten damit nicht nur an politischen Fragen, sondern auch am Sport nachvollzogen werden.

\section{Europäische Akteure im Sport}

Matthias Guetts Vortrag beschäftigte sich mit dem sportpolitischen Engagement des Europarates. Der Europarat habe 47 Mitgliedstaaten und repräsentiere rund 800 Millionen Menschen. Seine Aufgaben bestünden in einem engen Zusammenschluss seiner Mitglieder, der Förderung von Menschenrechten, Demokratie, Rechtsstaatlichkeit und der kulturellen Vielfalt seiner Mitglieder. Sport gehöre ebenfalls zu den verschiedenen Themen, mit denen sich der Europarat beschäftige. 1966 hätten die Aktivitäten des Europarats im Sportbereich mit einem ersten Beschluss zum Sport begonnen. Ein Markenzeichen der Aktivitäten stelle die Verknüpfung von staatlichen Akteuren mit Nichtregierungsorganisationen dar.

Die Verabschiedung der „Europäischen Charta über Sport für alle“" von 1976 und deren Überarbeitung im Jahr 1992 zu einer „Europäischen Sportcharta“ unter Ergänzung eines Ethikkodex seien Meilensteine der Aktivitäten des Europarats gewesen. 1985 sei eine Konvention über Gewalttätigkeit und Fehlverhalten von Zuschauern bei Sportveranstaltungen verabschiedet worden und 1989 eine Anti-DopingKonvention.

Der Europarat sei zusammen mit der Europäischen Union ein unverzichtbarer Partner zum Ansto $ß$ und zur Implementierung wichtiger Beschlüsse auf sportpolitischer Ebene. Der Europarat verfüge über mehr Mitglieder als die Europäische Union, sodass seine Beschlüsse für praktisch alle europäischen Staaten Geltung hätten.

Die Rolle der deutschen Bundesländer und der Sportministerkonferenz in der Sportpolitik beleuchtete Stefan Christmann. Den rechtlichen Rahmen der Zusammenarbeit der Bundes- und der Länderebene über Angelegenheiten der Europäischen Union bilde Art. 23 des Grundgesetzes. Kern der Zusammenarbeit stelle die Pflicht der Bundesregierung dar, die Länder über die Politiken der Europäischen Union zu informieren. 
Der Einfluss von sportpolitischen Maßnahmen der Europäischen Union auf die Länder müsse in jedem Einzelfall bewertet werden. Dies bedeute damit viel Arbeit für die Vertreter der Länder: Jede sportpolitische Maßnahme der Europäischen Union müsse hinsichtlich ihres genauen Einflusses auf die Länder geprüft werden.

Hans Bruyninckx steuerte einige grundlegende Gedanken über die europäische Sportpolitik bei. Sport bewege sich immer stärker von der „self-governance“" zur „,network-governance“, das heißt Sport regele sich nicht länger selbst ohne äußere politische Einflüsse. Globalisierung und Kommerzialisierung seien die treibenden Kräfte dieser Entwicklung. Negative Effekte von Globalisierung und Kommerzialisierung bestünden in Wettabsprachen, Korruption, Doping oder Geldwäsche.

Die negativen Seiten des Sports würden durch Staaten wie die Schweiz unterstützt. Sie verfüge über fünfzig Organisationen im Sportbereich und dies aus gutem Grund. Die Eidgenossenschaft sei spezialisiert, dubiosen Geldflüssen einen sicheren Hafen zu bieten. Das Justizsystem verfüge über wenige rechtliche Regelungen, um illegal erworbenes Geld zu verfolgen und zu beschlagnahmen.

Weiterhin führte Bruyninckx aus, dass es kein einheitliches „Europa“ im Sport gebe. Verschiedene Sportwettbewerbe umfassten verschiedene Staaten. Genauso würden verschiedene internationale Organisationen, wie die Europäische Union oder der Europarat, unterschiedliche Staaten umfassen. Israel sei beispielsweise ein Teil der Fußball-Europameisterschaft.

Die Bedeutung des Sports zeigt sich schließlich nach Ansicht von Bruyninckx darin, dass über die Fußball-Europameisterschaft 2012 in den Medien mehr berichtet wurde als über die Europäische Union, die sich in einer ihrer größten Krisen befände.
Sport als Katalysator politischer und gesellschaftlicher Trends

In der abendlichen Podiumsdiskussion wurde über das Verhältnis von Sport und Politik am Beispiel der Fußball-Europameisterschaft in Polen und der Ukraine gesprochen. Nach Ansicht von Olaf Sundermeyer verstärkten internationale Sportereignisse bereits bestehende politische Trends und Entwicklungen in den Gastgeberländern. Dies habe sich bei den Olympischen Spielen 2008 in Peking und bei der Fußball-Europameisterschaft 2012 gezeigt. Die autoritäre Herrschaft in China sei gestärkt aus der Ausrichtung der Olympischen Spiele hervorgegangen. Die positive soziale, politische und wirtschaftliche Entwicklung Polens werde ebenfalls durch die Fußball-Europameisterschaft gefördert.

In der Ukraine seien ebenso die bestehenden Entwicklungen hin zu einem autoritäreren Staat bestätigt worden: Die Austragung der Fußball-Europameisterschaft in der Ukraine habe die oligarchischen Strukturen gestärkt. Die „Respect“-Kampagne der Union des Associations Européennes de Football (UEFA) gegen Diskriminierung und Rassismus in der Ukraine werde keine bleibende Wirkung haben. Vermutlich würden die rassistischen Fans nach der Fußball-Europameisterschaft erneut die ukrainischen Stadien dominieren.

Im Sport sei nach Ansicht von Folker Hellmund ein größeres Bewusstsein für dessen gesellschaftliche und politische Bedeutung entstanden und die Sportler seien in Bezug auf die politischen Verhältnisse in den Gastgeberländern kritischer geworden. Dies habe sich zum Beispiel an Philipp Lahms Kritik an den politischen Verhältnissen in der Ukraine gezeigt.

Thomas Horky erwähnte, dass die kritische Berichterstattung im Sport, beispielsweise über die Ukraine, nur in einigen Medien zu finden sei. Deutschlandfunk, Deutschlandradio, die Frankfurter Allgemeine Zeitung und die Süddeutsche Zeitung gehörten zu den positiven Ausnahmen. Die mediale Berichterstattung 
zeichne sich insgesamt durch einen ereignisbezogenen und punktuellen Journalismus aus. Kaum jemand berichte über Südafrika und leere Fußballstadien, weil die Fußball-Weltmeisterschaft vorbei sei. Horky berichtete weiter, dass die Fußball-Europameisterschaft in den Medien ein quantitativer Erfolg sei. Die $\mathrm{Zu}-$ schauerzahlen seien sehr hoch. Die Qualität der Berichterstattung habe hingegen eher gelitten (ein Beispiel ist die Berichterstattung des ZDF zur Fußball-Europameisterschaft 2012 vom Strand der Insel Usedom).

\section{Sportliche Herausforderungen:}

Arbeitsrechtliche Konflikte, Doping und zu wenig Geld

Walfried König referierte über die Auswirkungen des Vertrags von Lissabon auf den Sport. Er begann mit einigen grundsätzlichen Gedanken über die Förderung des Sports durch das europäische Primärrecht. Das Kapitel „Sozialpolitik" des AEUV enthalte auch die Sportförderung. So lege Art. 165 AEUV zwar den Sport als Ziel der Europäischen Union fest, aber dies bedeute noch nicht, dass Sport automatisch gefördert werde.

Weiterhin erläuterte König rechtliche Aspekte der europäischen Sportpolitik. So würden Sportvereine von der Europäischen Union als Betriebe klassifiziert und dies bedeute, dass sozialpolitische Regelungen der Europäischen Union sich auf diese anwenden ließen. Betriebliche Mitbestimmung und Schutzklauseln der Arbeitnehmer würden damit ebenfalls Anwendung auf Sportvereine finden. Kinderarbeit, Arbeit in den Abendstunden, die Gefährdungslage von Rennstrecken oder die freie Wahl des Physiotherapeuten seien Beispiele, bei denen die Europäische Union sozialpolitische Regelungen treffen könnte.

Etwa 800.000 Menschen in der Europäischen Union hätten einen hauptberuflichen Arbeitsplatz im Sport und etwa 15 Millionen seien insgesamt im Sportbereich engagiert. Die Arbeitnehmer im Sport und die Sportler verfügten inzwischen über ein hohes Selbstbewusstsein und forderten ihre Rechte als Arbeitnehmer ein. Eine Konsequenz sei die Bildung von Interessenvertretungen, wie beispielsweise Vereinigungen von Sportlern. Viele Arbeitsverträge im Sportbereich würden arbeitsrechtlichen Konflikten vor Gericht nicht standhalten. Viele der Verträge umfassten keine sportspezifischen Regelungen, wie zu Arbeitszeiten am Abend oder am Wochenende. Ein großes Defizit sei, dass es in Deutschland bislang keine Dachverbände als Interessenvertretung der Arbeitgeber und Arbeitnehmer im Sport gebe.

Dopingpolitik war das Thema des Vortrags von Henk Erik Meier. Auf der Ebene der Sportler und der nationalen Sportverbände existiere ein Gefangenendilemma. Beide Akteure müssten dopen beziehungsweise Doping zulassen, weil sie nur so konkurrenzfähig seien. Weiterhin gebe es ein Ressourcendilemma und ein Organisationsdilemma. Antidopingmaßnahmen seien teuer und es stelle sich immer die Frage, woher das Geld für Antidopingmaßnahmen komme. Das Ressourcendilemma gelte für alle Staaten, im Besonderen für Entwicklungsländer.

Eine weitere Schwierigkeit bestehe in einem komplexen Verhältnis zwischen staatlichem Recht und Sportverbandsrecht. So stelle sich die Frage der Strafwürdigkeit von Doping nach staatlichem Recht und nach Verbandsrecht. Im Verbandsrecht führten Antidopingmaßnahmen meist zu einem Berufsverbot für gedopte Sportler.

Durchbrüche in der Dopingpolitik auf internationaler Ebene seien die Etablierung der World Anti-Doping Agency (WADA) im Jahr 1999 und des WADA-Codes im Jahr 2003 gewesen. Der WADA-Code harmonisierte die weltweiten Regelungen im Bereich des Kampfes gegen Doping. Völkerrechtlich abgesichert seien beide durch die „International Convention Against Doping in Sport" der United Nations Educational, Scientific and Cultural Organization (UNESCO) aus dem Jahr 2005. Das Internationale Olympische Komitee setze die Regelungen durch und könne als Sanktion das 
Verbot der Teilnahme an Olympischen Spielen verhängen.

Die Europäische Union beginne schließlich mit einer Antidopingpolitik, bislang aber mit eher bescheidenem Erfolg. Sie besitze keine Budgethoheit im Bereich des Sports und es mangele damit an finanziellen Mitteln für Antidopingmaßnahmen. Der Europarat habe hingegen im Bereich der Antidopingpolitik eine Vorreiterrolle gespielt. Die Europäische Union habe aber einen positiven und maßgeblichen Einfluss auf die Struktur der WADA genommen. Bestehende Probleme aus EU-Sicht bestünden in einer Unterrepräsentanz der EUStaaten im Aufsichtsrat der WADA, obwohl EU-Mitgliedstaaten etwa die Hälfte des Budgets bestreiten.

Roland Naul sprach über die „EU Physical Activity Guidelines“. Im Jahr 2004 habe es eine Studie der Europäischen Union über die Lebensverhältnisse junger Menschen gegeben. Diese habe als Anlass gedient, um verstärkte Aktivitäten der Europäischen Union im Bereich des Sports zu initiieren. Im Jahr 2007 habe die Europäische Union ein Weißbuch Sport veröffentlicht und zum ersten Mal sei die soziale und gesellschaftspolitische Rolle des Sports offiziell anerkannt und beschrieben worden, beispielsweise für die Bildung, die physische Aktivität der Bürger und im Kampf gegen Rassismus.

Sport, Gesundheit und Bildung bildeten die drei Hauptbereiche der „EU Physical Activities Guidelines". Die EU-Richtlinien seien im Jahr 2008 verabschiedet worden. Nur einige EU-Staaten hätten vorher über nationale Richtlinien verfügt. Die Umsetzung der Richtlinien könne nur durch eine Zusammenarbeit der Akteure auf den verschiedenen Ebenen erreicht werden. Ein Beispiel für die Ziele in den Richtlinien seien gesundheits- und bewegungsfreundlichere Arbeitsplätze. Es werde auch ein Monitoringsystem zur Überwachung der Umsetzung der Richtlinien entwickelt. Sie seien in Deutschland kaum bekannt. Der Ansatz der Verbindung von Gesundheit, Sport und Bil- dung fände bislang keine Entsprechung in der Politik des Bundes und der Bundesländer in Deutschland.

Daniel Dickmann referierte über die finanzielle Dimension europäischer Sportpolitik. Finanzierungsquellen des Sports in der gesamten Europäischen Union kämen überwiegend aus Privathaushalten (55 Prozent) und der ehrenamtlichen Tätigkeit (15 Prozent) (Zahlen aus 2008). Ausgaben für Sportartikel gehörten aber zu diesem Bereich, sodass die Zahlen mit Vorsicht zu betrachten seien.

Die Europäische Union finanziere nur einen sehr kleinen Teil des Sports. In den Jahren 2009 bis 2012 habe die Europäische Union nur wenige Millionen Euro pro Jahr für die Sportförderung ausgegeben. Für den Zeitraum 2014 bis 2020 stünden insgesamt 238 Millionen Euro für die Sportförderung zur Verfügung, also 34 Millionen Euro pro Jahr. Die Förderung finde über das Programm „Erasmus für Alle“ statt. Das Ziel bestehe in der Schaffung einer europäischen Dimension im Sport. Das Programm wende sich sowohl an öffentliche als auch an zivilgesellschaftliche Einrichtungen. Sport werde aber im Zeitrahmen 2014 bis 2020 auch indirekt, durch sogenanntes ,mainstreaming“, gefördert. Das bedeute, die Förderung des Sports werde in viele Programme der Europäischen Union integriert.

Die abschließende These von Dickmann lautete: Je weiter sich die Europäische Union einem eigenständigen Sportförderprogramm nähere, desto größer werde die Gefahr der Marginalisierung des Sports bei der Vergabe von Projekten aus verwandten Förderprogrammen. Es bestehe die Gefahr, dass bei verwandten Förderprogrammen Anträge auf Förderung sportspezifischer Projekte mit dem Verweis auf ein eigenständiges Sportförderprogramm der Europäischen Union abgewiesen würden.

Schließlich fehle eine sogenannte horizontale Klausel im Primärrecht der Union, die dazu führe, dass Sport in allen Politikbereichen mitgedacht werden müsse, wie es beispielsweise im Gesundheitsbereich der Fall sei. 


\section{Internationalisierung, Medialisierung und Diskriminierung im Fußball}

Der Vortrag von Benjamin Legrand beschäftigte sich mit der Gründung und Entwicklung der Fußball-Europameisterschaften. Die europäischen Verbände dominierten nach dem Ende des Zweiten Weltkrieges nicht mehr länger die Fédération Internationale de Football Association (FIFA). Dies habe unter anderem zur Gründung der UEFA 1954 geführt. Die Etablierung einer Fußball-Europameisterschaft durch die UEFA im Jahr 1960 sei anfangs auf wenig Interesse gestoßen. Das Interesse der Zuschauer sei erst seit den 1980er Jahren stark gestiegen und das Fernsehen habe begonnen die Spiele zu zeigen. Die Kapazitätsauslastung der Stadien sei ebenfalls auf fast 100 Prozent gestiegen. In der Frühphase, beispielsweise noch im Jahr 1980, sei die Auslastung bei Spielen, in denen die Gastgebermannschaft Italien nicht gespielt hätte, teilweise gering gewesen (etwas über 20 Prozent).

Die Haupteinnahmen der UEFA kämen inzwischen aus dem Verkauf der TV-Übertragungsrechte. Die Fußball-Europameisterschaften bildeten insgesamt ein kurzes Zeitfenster für eine europäische Öffentlichkeit, da ein großes mediales und öffentliches Interesse für dieses Ereignis bestehe.

Jörg-Uwe Nieland sprach über internationale Fußballturniere als Wegbereiter transnationaler Sportpolitik. Mediale Sportereignisse zeichneten sich meist durch eine hohe Dramatik und Symbolik aus. Die Fußball-Weltmeisterschaft nehme in Deutschland, England, Frankreich und Italien den Platz als beliebtestes Sportereignis ein. Im Jahr 2010 seien unter den zehn am meisten gesehenen Sportereignissen in Deutschland neun Spiele der FußballWeltmeisterschaft gewesen. Das zehnte Spiel sei das Qualifikationsspiel zur Fußball-Europameisterschaft Deutschland-Türkei gewesen. In einer Befragung von Türken in Deutschland anlässlich der Fußball-Weltmeisterschaft 2010 habe Nieland herausgefunden, dass sich die Türken vor allem über Mesut Özil unterhalten haben und er auch als der sympathischste Spieler betrachtet wurde sowie als der zweitbeste nach Lionel Messi. In über einem Drittel der Berichte über das EM-Qualifikationsspiel Deutschland-Türkei sei das Thema Integration erwähnt worden. Dies treffe vor allem, aber nicht nur, für den Sportteil zu. Dies gelte sowohl für deutsche als auch für türkische Zeitungen.

Die Internationalisierung des Sports zeige sich auch in der Anzahl internationaler Spieler in nationalen Fußballigen. In England spielten die meisten internationalen Spieler. In Europa spielten die meisten internationalen Spieler beim FC Barcelona (81 Prozent) und in Deutschland beim FC Bayern München (zwei Drittel der Spieler). Hindernisse für die Europäisierung des Sports lägen in der weiterhin bestehenden starken nationalen Identifikation und einer teilweise national gefärbten Berichterstattung, wie geäußerten Ängsten vor einer Überfremdung von Mannschaften.

Gerd Dembowski referierte über Fans im Sport. Bei der Fußball-Europameisterschaft 2012 seien zum ersten Mal Beobachter für antidiskriminierendes Verhalten in den Stadien eingesetzt worden. Diese überwachten die Spieler und die Zuschauer, um ein mögliches diskriminierendes Verhalten zu dokumentieren. Bei der Registrierung diskriminierenden Verhaltens würden Sanktionen verhängt. Die Einführung dieser Maßnahmen bei der Fußball-Europameisterschaft 2012 habe nichts mit den Gastgeberländern Polen und der Ukraine zu tun gehabt.

Der Deutsche Fußballbund nehme eine besondere Stellung unter den nationalen Verbänden ein, weil er sich als einziger Verband flächendeckend Fanprojekte leiste. Insgesamt gebe es eine positive Entwicklung bei den deutschen Fans, die sich inzwischen weniger diskriminierend in ihren Fangesängen und anderen Äußerungen verhielten. Das gelte aber nicht unbedingt für Fußballfans anderer Nationen.

Fußballfans stellten eine Gruppe dar, die aus sich selbst heraus Engagement gegen diskri- 
minierendes Verhalten entwickelt habe. Fußball in den 1980er Jahren sei ,asozial“ gewesen. Das Verhalten vieler Fans könne als dumpf beschrieben werden. Erst später habe es eine „Elffreundisierung“ des Fußballs, das heißt eine anspruchsvollere Berichterstattung über Fußball und eine Erweiterung des Kreises der Fans auf intellektuellere Schichten der Bevölkerung gegeben. Dies erkläre unter anderem, warum die Toleranz im Fußball gestiegen und die Gewalt gesunken sei.

Fußball bleibe aber eine Domäne „hegemonialer Männlichkeit": Teilweise koexistiere Toleranz von Fans gegenüber beispielsweise der Hautfarbe von Fußballspielern mit Homophobie. Im Fußball gebe es außerdem eine klare Trennung zwischen „Wir“ und den „Anderen“. In der Regel seien Fußballfans nur Fans eines Vereins. Bei Musikbands könne man dieses Phänomen beispielsweise nicht beobachten. Dies verstärke ein ab- und ausgrenzendes Verhalten von Fans. Die Tendenz auf europäischer Ebene laufe stärker auf den Ansatz der Bestrafung bei diskriminierendem Verhalten hinaus. Die Lösung liege aber mehr in der Förderung sozialer Projekte.
Fazit: Die ewige Frage der Zukunft Europas und der europäischen Sportpolitik

Die Tagung hat nach Ansicht von Jürgen Mittag ein breites Themenspektrum abgedeckt. Sportpolitik gehöre in die Politikwissenschaft. Allerdings müssten die Impulse aus der Rechts- und Wirtschaftswissenschaft aufgegriffen und ernst genommen werden. Das $\mathrm{Zu}$ sammenspiel unterschiedlicher Akteure und verschiedener Politikebenen präge die europäische Sportpolitik. Der nationalstaatliche und europäische Einfluss auf den Sport wachse.

Die Tagung habe sich um eine Bestandsaufnahme der europäischen Sportpolitik bemüht. Dabei seien verschiedene theoretische Ansätze verwendet und kombiniert worden. Eine übergeordnete Frage bleibe: Wohin entwickelt sich die europäische Sportpolitik? Diese Frage schließe nahtlos an die dominante Frage der Europawissenschaft an: Wohin entwickelt sich Europa? 